\title{
Microscopic structure of shocks in one conservation laws
}

\author{
by \\ Fraydoun REZAKHANLOU ${ }^{(1)}$ \\ Department of Mathematics, University of California, \\ Berkeley, California 94720, U.S.A.
}

\begin{abstract}
We show that in one conservation laws, the disturbances are propagated along the characteristic lines and shocks. We use this to investigate the macroscopic behavior of the second class particles in stochastic models such as asymmetric simple exclusion and zero range processes, in one space dimension. We show that a second class particle will follow the characteristic lines and shocks of the hydrodynamic equation.
\end{abstract}

\section{INTRODUCTION}

An outstanding problem in statistical mechanics is to establish the connection between the microscopic structure of a fluid and its macroscopic behavior. Perhaps the most celebrated problem in this context is the derivation of Euler's equations from the small scale dynamics governed by Newton's second law. Euler's equations can also be obtained through purely macroscopic considerations. One can formally derive these equations employing the conservation of mass, momentum and energy.

We can simplify the problem above by considering other models with only one conservation law, one such being that of particles with simple

( $\left.{ }^{1}\right)$ Research supported by National Science Foundation grant DMS-9208490. 
exclusions. Although this model is simpler, it retains many aspects of the original problem.

An important aspect of one conservation law with nonlinear flux is the development of discontinuities in its solutions, physically corresponding to the occurrence of shocks in fluids. The main objective of this paper is to investigate the microscopic structure of shocks in one conservation law.

The simple exclusion process (SEP) is a continuous time particle system in which particles move as random walks on a one-dimensional lattice but are excluded from occupying the same site. The precise definition of SEP will be given in the next section.

The Law of Large Numbers for random walks suggests scaling space by a small factor of order $L^{-1}$, and speeding time by a large factor of order $L$. If $\rho(x, t)$ denotes the macroscopic particle density, it is known that $\rho$ satisfies the hydrodynamic equation

$$
\rho_{t}+\gamma h(\rho)_{x}=0
$$

where $\gamma$ is a constant denoting the stochastic mean of the underlying random walk, and $h(\rho)=\rho(1-\rho)$. See for example [13] and the references therein.

It is well known that if $h$ is nonlinear, equation (1.1) does not in general possess globally defined smooth solutions. It is therefore necessary to interpret (1.1) in the distributional sense. Since there are infinitely many distributional solutions to (1.1) that share the same initial condition, some additional conditions are needed to ensure uniqueness. We say $\rho$ is an entropy solution if it satisfies

$$
\phi(\rho)_{t}+\gamma q(\rho)_{x} \leq 0
$$

for every entropy pair $(\phi, q)$ with $\phi$ convex and $q$ satisfying $\phi^{\prime} h^{\prime}=q^{\prime}$.

There is a simple recipe for constructing entropy solutions of (1.1), providing $h$ is either strictly convex or strictly concave. Let us assume $\gamma h$ is strictly convex and continuously differentiable.

Let $y=y(x, t)$ denote a point at which the minimum of

$$
t L\left(\frac{x-y}{t}\right)+w_{0}(y)
$$

is attained, where $L$ is the convex conjugate of $\gamma h$

$$
L(q)=\sup _{\rho}(\rho q-\gamma h(\rho))
$$


$w_{0}$ is given by

$$
w_{0}(y)=\int_{-\infty}^{y} \rho_{0}(z) d z
$$

and $\rho_{0}$ denotes the initial density.

Then, according to the Lax-Oleinik formula [9], $\rho$ can be defined by

$$
\gamma h^{\prime}(\rho(x, t))=\frac{x-y(x, t)}{t}
$$

It turns out that for each positive $t, y(x, t)$ is nondecreasing in $x$. We denote the inverse of $y(\cdot, t)$ by $x(\cdot, t)$.

One of the main results of this paper characterizes $x(a, \cdot)$ as the trajectory along which the disturbances in the density propagate. More precisely, let $\rho_{0, \delta}(\cdot)$ be an initial density which is different from $\rho_{0}(\cdot)$ only in a $\delta$ neighborhood of the point $a$. If $\rho^{\delta}(x, t)$ denotes the unique entropy solution of (1.1) with the initial density $\rho_{0, \delta}$, then $\rho^{\delta}(x, t)$ is different from $\rho(x, t)$ only in a small neighborhood of $x(a, t)$.

We also identify $x(t)=x(a, t)$ to be the generalized characteristic of (1.1) emanating from $a$. Generalized characteristics were initiated by Dafermos [2] as solutions to the ODE

$$
\left\{\begin{array}{l}
\frac{d x}{d t}=\gamma h^{\prime}(\rho(x, t)) \\
x(0)=a
\end{array}\right.
$$

Since $\rho$ is not continuous, (1.6) is understood in the Filippov sense [7]: an absolutely continuous function $x$ is a solution if for almost all $t, \frac{d x}{d t}$ is between the essential infimum and the essential supremum of $\gamma h^{\prime}(\rho)$ evaluated at the point $(x(t), t)$.

It can be shown that the generalized characteristics are either classical characteristics or shocks. In the former case $\rho$ is continuous at $(x(t), t)$ whereas in the latter case $\rho$ is discontinuous at $(x(t), t)$.

For nonconvex $h$, the identification of the propagation of disturbances with the generalized characteristics remains open.

It is believed that, at least for piecewise constant densities, second class particles follow either shocks or characteristic lines. To define a second class particle, we take two systems of particles that are different initially at a single site - one of the systems contains an additional particle. Both systems evolve according to SEP and it is possible to couple them in such 
a way that at later times, the discrepancy between the two will occur at a single site. This site is the location of the additional particle known as the second class particle.

Ferrari [4] shows that if a second class particle is initially added to the site $[a L]$ (the integer part of $a L$ ), then $\frac{1}{L}$ times the location of the second class particle at time $t L$ converges to $x(a, t)$, provided that the initial density is constant on the left and right sides of the origin (Riemann solutions). We will show that Ferrari's theorem holds even if the density is not a Riemann solution. In fact our results are not restricted to SEP. They also apply to zero range processes with concave rate functions. See the next section for details.

An important problem in this context is the fluctuation of the position of the second class particle around the trajectory of $x(a, t)$. A better approximation for the location of the second class particle is conjectured to be

$$
x(a, t)+\frac{1}{\sqrt{L}} \frac{1}{\rho^{+}-\rho^{-}} \int_{y^{-}(x(t), t)}^{y^{+}(x(t), t)} \xi_{0}(z) d z
$$

where $\rho^{ \pm}=\rho^{ \pm}(x(t), t)$ are the left and right limits of $\rho$ at $(x(t), t)$, $y^{ \pm}(x, t)$ are the largest and smallest value $y$ at which the minimum of (1.3) is attained, and $\xi_{0}$ denotes the random noise of the initial density. It is not hard to see that the second term in (1.7) is a Gaussian process where in the case of SEP its variance equals

$$
\frac{1}{L} \frac{1}{\left(\rho^{+}-\rho^{-}\right)^{2}} \int_{y^{-}(x(t), t)}^{y^{+}(x(t), t)} \rho_{0}(z)\left(1-\rho_{0}(z)\right) d z .
$$

So far (1.7) has been established for SEP in the case of Riemann solutions ([4-6], [8], [15]). In the last section we will give a formal derivation of (1.7) for general initial data. See also Spohn [14].

The organization of this paper is as follows: $\S 2$ is devoted to the statement of our main results. In $\S 3$ we study the propagation of disturbances and its relation with generalized characteristics. The proof of the Law of Large Numbers for second class particles will be given in $\S 4$. A formal derivation of the formula (1.7) will be given in the last section.

\section{NOTATION AND MAIN RESULTS}

The primary purpose of this section is the statement of the main results. This section is divided into three parts. In the first part we define SEP, zero range processes and state a theorem concerning their hydrodynamical 
behavior. In the second part we discuss the Lax-Oleinik formula and the Filippov solution. The main results of this paper will be presented in the last part.

\section{Part 1}

We start with the definition of the so-called processus misanthrope that includes the SEP.

Let $p(1)$ and $p(-1)$ be two nonnegative numbers with $p(1) \leq p(-1)$ and $p(1)+p(-1)=1$. For notational convenience we define $p(z)$ for all $z \in \mathbb{Z}$ and set $p(z)=0$ for $z \neq 1,-1$.

Let $b: \mathbb{N} \times \mathbb{N} \rightarrow[0, \infty)$ be a bounded function with the following properties:

(i) $b(0, \cdot) \equiv 0$

(ii) $n \mapsto b(n, m)$ is a nondecreasing function for each $m$

(iii) $m \mapsto b(n, m)$ is a nonincreasing function for each $n$

Given $p$ and $b$ we define $\left(\eta_{t}(u): u \in \mathbb{Z}\right)$ as the unique Feller process with state space $E=\mathbb{N}^{\mathbb{Z}}$ and the infinitesimal generator $\mathcal{L}$, where $\mathcal{L}$ acting on cylinder functions, is defined by

$$
\mathcal{L} f(\eta)=\sum_{u, v} p(v-u) b(\eta(u), \eta(v))\left(f\left(\eta^{u v}\right)-f(\eta)\right)
$$

where

$$
\eta^{u v}(z)= \begin{cases}\eta(u)-1 & \text { if } z=u \\ \eta(v)+1 & \text { if } z=v \\ \eta(z) & \text { if } z \neq u, v\end{cases}
$$

provided $\eta(u) \geq 1$ and $u \neq v ; \eta^{u v}=\eta$ otherwise.

Some restrictions on $b$ are needed to ensure that the invariant measures for the process $\eta$ are of simple form. We will formulate conditions that imply product measures are invariant.

Let $g: \mathbb{N} \rightarrow[0, \infty)$ be a given bounded nondecreasing function with $g(0)=0$. For such $g$, and any given $\lambda \in\left[0, \sup _{k} g(k)\right)$ we define a probability measure $\Theta_{\lambda}$ on $\mathbb{N}$ by

$$
\Theta_{\lambda}(n)= \begin{cases}\frac{1}{Z(\lambda)} \frac{\lambda^{n}}{g(1) \ldots g(n)} & \text { if } n \neq 0 \\ \frac{1}{Z(\lambda)} & \text { if } n=0\end{cases}
$$


where $Z(\lambda)$ is the normalizing factor. Set

$$
\varphi(\lambda)=\sum_{n=1}^{\infty} n \Theta_{\lambda}(n) .
$$

Then

$$
\varphi:\left[0, \sup _{k} g(k)\right) \rightarrow[0, \infty)
$$

is strictly increasing, and

$$
\lim _{\lambda \rightarrow \sup _{k} g(k)} \varphi(\lambda)=+\infty
$$

The inverse of $\varphi(\cdot)$ will be denoted by $\lambda(\cdot)$ and let $\Theta^{\rho}=\Theta_{\lambda(\rho)}$. The probability measure $\nu^{\rho}$ is obtained by taking the product of $\Theta^{\rho}$,

$$
\nu^{\rho}(d \eta)=\prod_{u \in \mathbb{Z}^{d}} \Theta^{\rho}(d \eta(u))
$$

so that $\nu^{\rho}(\eta(u)=k)=\Theta^{\rho}(k)$. We certainly have

$$
\begin{aligned}
& \int \eta(0) \nu^{\rho}(d \eta)=\rho \\
& \int g(\eta(0)) \nu^{\rho}(d \eta)=\lambda(\rho) .
\end{aligned}
$$

For a given $b$ as in (2.1), we assume that there exists a bounded nondecreasing $g$ such that

$$
b(n, m-1) g(m)=b(m, n-1) g(n)
$$

for $n, m \geq 1$. We also assume

$$
b(n, m)-b(m, n)=b(n, 0)-b(m, 0)
$$

for $n, m \geq 0$. Conditions (2.5) and (2.6) imply that $\nu^{\rho}$ are invariant with respect to $\mathcal{L}$.

If we choose

$$
b(n, m)= \begin{cases}1 & \text { if } n=1, m=0 \\ 0 & \text { otherwise }\end{cases}
$$

and restricting the process $\eta$ to $\{0,1\}^{\mathbb{Z}}$, we obtain the SEP. In this case there is at most one particle per site. For SEP, the invariant measures are 
$\left\{\nu^{\rho}: \rho \in[0,1]\right\}$, where each $\nu^{\rho}$ is a product measure with marginals $\Theta^{\rho}$ where

$$
\Theta^{\rho}(k)=\left\{\begin{array}{lll}
\rho & \text { if } & k=1 \\
1-\rho & \text { if } & k=0
\end{array} .\right.
$$

If we choose $b(n, m)=g(n)$ both (2.5) and (2.6) are satisfied and the resulting process is called a zero range process (ZRP). In this case the rate of jump from $u$ to $v$ does not depend on the occupation number $\eta(v)$.

We now describe the kind of initial distribution that we will consider for the process $\eta_{t}$.

Notation 2.1. - Let $\mu_{L}$ be a sequence of probability measures on $E$ and let $\rho_{0}: \mathbb{R} \rightarrow[0, \infty)$ be a bounded integrable function. We then write $\mu_{L} \sim \rho_{0}$ if the following conditions hold:

(a) $\mu_{L}$ is a product measure

(b) there exists a sequence of constants $\rho_{u, L}$ such that

$$
\begin{gathered}
\mu_{L}(\eta(u)=k)=\Theta^{\rho_{u}, L}(k) \quad u \in \mathbb{Z}, k \in \mathbb{N} \\
\lim _{L \rightarrow \infty} \int_{|x| \leq \ell}\left|\rho_{[x L], L}-\rho_{0}(x)\right| d x=0
\end{gathered}
$$

for every positive $\ell$. ( $[x L]$ denotes the integer part of $x L$.)

Note that if $\rho_{0}$ is continuous, we may choose $\rho_{u, L}=\rho_{0}\left(\frac{u}{L}\right)$ for every $u \in \mathbb{Z}$.

We define the constant $\gamma$ as the mean of $p$ :

$$
\gamma=p(1)-p(-1)
$$

and the scalar valued function $h$ as the average of $b$ with respect to the invariant measure $\nu^{\rho}$ :

$$
h(\rho)=\int b(\eta(u), \eta(v)) \nu^{\rho}(d \eta), \quad u \neq v .
$$

Since $\nu^{\rho}$ is a translation invariant product measure, the right side of (2.8) is independent of $u$ and $v$.

In the case of SEP, $h(\rho)=\rho(1-\rho)$ and in the case of ZRP, $h(\rho)=\lambda(\rho)$ (see (2.4)).

Let $\rho(x, t)$ denote the unique entropy solution of

$$
\left\{\begin{array}{l}
\rho_{t}+\gamma h(\rho)_{x}=0 \\
\rho(x, 0)=\rho_{0}(x)
\end{array}\right.
$$


More precisely, (1.2) holds for every entropy pair and

$$
\lim _{t \rightarrow 0} \int_{|x| \leq \ell}\left|\rho(x, t)-\rho_{0}(x)\right| d x=0
$$

for every positive $\ell$.

Let $P_{L}$ denote the distribution $\eta$ where $\eta_{0}$ is distributed according to $\mu_{L}$. In [13] we proved

THEOREM 2.2. - Suppose $\mu_{L} \sim \rho_{0}$ for some $\rho_{0} \in L^{1} \cap L^{\infty}$. Then for every bounded continuous $J$, and every $t \geq 0$,

$$
\lim _{L \rightarrow \infty} \int\left|\frac{1}{L} \sum_{u} J\left(\frac{u}{L}\right) \eta_{t L}(u)-\int J(x) \rho(x, t) d x\right| P_{L}(d \eta)=0 .
$$

An important implication of the monotonicity assumption (2.1) is the monotonicity of the process $\eta_{t}$. For this we first define the following partial order on $E: \eta \leq \zeta$ if $\eta(u) \leq \zeta(u)$ for all $u \in \mathbb{Z}$. It is possible to construct a coupled process $\left(\eta_{t}, \zeta_{t}\right)$ on $E \times E$ such that the evolutions of $\eta_{t}$ and $\zeta_{t}$ are governed by $\mathcal{L}$, and if initially $\eta_{0} \leq \zeta_{0}$ then $\eta_{t} \leq \zeta_{t}$ for all $t$. The generator of $\left(\eta_{t}, \zeta_{t}\right)$ is given by $\widetilde{\mathcal{L}}$, where

$$
\begin{aligned}
\widetilde{\mathcal{L}} f(\eta, \zeta) & =\sum_{u, v} p(v-u)(b(\eta(u), \eta(v)) \\
& \wedge b(\zeta(u), \zeta(v)))\left(f\left(\eta^{u v}, \zeta^{u v}\right)-f(\eta, \zeta)\right) \\
& +\sum_{u, v} p(v-u)(b(\eta(u), \eta(v)) \\
& -b(\eta(u), \eta(v)) \wedge b(\zeta(u), \zeta(v)))\left(f\left(\eta^{u v}, \zeta\right)-f(\eta, \zeta)\right) \\
& +\sum_{u, v} p(v-u)(b(\zeta(u), \zeta(v)) \\
& -b(\eta(u), \eta(v)) \wedge b(\zeta(u), \zeta(v)))\left(f\left(\eta, \zeta^{u v}\right)-f(\eta, \zeta)\right)
\end{aligned}
$$

In particular, if

$$
\begin{aligned}
\zeta_{0}(u) & =\eta_{0}(u) \quad \text { for } u \neq x_{0}, \text { and } \\
\zeta_{0}\left(x_{0}\right) & =\eta_{0}\left(x_{0}\right)+1
\end{aligned}
$$

for some $x_{0}$, then this situation persists at later times. In other words, there exists a site $x_{t}$ so that

$$
\begin{aligned}
\zeta_{t}(u) & =\eta_{t}(u) \quad \text { for } u \neq x_{t}, \text { and } \\
\zeta_{t}\left(x_{t}\right) & =\eta_{t}\left(x_{t}\right)+1
\end{aligned}
$$


It turns out that the process $\left(\eta_{t}, x_{t}\right)$ is also a Markov process and its infinitesimal generator is given by

$$
\begin{aligned}
\mathcal{A} f(\eta, x)= & \sum_{v \neq x, u} p(v-u) b(\eta(u), \eta(v))\left(f\left(\eta^{u v}, x\right)-f(\eta, x)\right) \\
+ & \sum_{k} p(k)(b(\eta(x)+1, \eta(x+k))-b(\eta(x), \eta(x+k))) \\
& (f(\eta, x+k)-f(\eta, x)) \\
+ & \sum_{k} p(k)(b(\eta(x-k), \eta(x))-b(\eta(x-k), \eta(x)+1)) \\
& \left(f\left(\eta^{x-k, x}, x-k\right)-f(\eta, x)\right) \\
+ & \sum_{k} p(k) b(\eta(x-k), \eta(x)+1)\left(f\left(\eta^{x-k, x}, x\right)-f(\eta, x)\right)
\end{aligned}
$$

The main goal of this paper is to study the asymptotic behavior of $\frac{1}{L} x_{t L}$ as $L$ goes to infinity.

\section{Part 2}

Throughout the paper we assume $h$ is nonlinear.

Assumption 2.3. $-h$ is either strictly convex or strictly concave, i.e. $h^{\prime \prime}(\rho) \neq 0$ for all $\rho$.

Under this assumption, $\rho(t, \cdot)$ can only have first kind discontinuities for every positive $t$. Let us assume $\gamma h$ is strictly convex.

For every positive $t$ define

$$
w(y, t)=\int_{-\infty}^{y} \rho(z, t) d z
$$

It is well known that $w$ solves the Hamilton-Jacobi PDE

$$
\left\{\begin{array}{l}
w_{t}+\gamma h\left(w_{y}\right)=0 \quad t>0 \\
w(y, 0)=w_{0}(y)
\end{array}\right.
$$

and in fact $w$ is given by Hopf's formula (see [9], [10]). More precisely if $0 \leq t_{1}<t_{2}$, then

$$
w\left(x, t_{2}\right)=\min _{y}\left(\left(t_{2}-t_{1}\right) L\left(\frac{x-y}{t_{2}-t_{1}}\right)+w\left(y, t_{1}\right)\right)
$$

where $L$ is the convex conjugate of $\gamma h$ (see (1.4)). 
Let $I\left(x ; t_{1}, t_{2}\right)$ denote the set of values $y$ at which the minimum in (2.13) is attained. Let $y^{+}\left(x ; t_{1}, t_{2}\right)$ and $y^{-}\left(x ; t_{1}, t_{2}\right)$ denote, respectively the largest and the smallest values in $I\left(x ; t_{1}, t_{2}\right)$. It is shown in [9] that $y^{ \pm}\left(x ; t_{1}, t_{2}\right)$ is nondecreasing in $x_{2}$. More precisely if $x_{1}<x_{2}$, then $y^{+}\left(x_{1} ; t_{1}, t_{2}\right) \leq y^{-}\left(x_{2} ; t_{1}, t_{2}\right)$. Furthermore we define

$$
\rho^{ \pm}\left(x, t_{2}\right)=G\left(\frac{x-y^{ \pm}\left(x ; t_{1}, t_{2}\right)}{t_{2}-t_{1}}\right), \quad t_{2}>t_{1}
$$

where $G=L^{\prime}$ (the derivative of $L$ ). Clearly $\rho^{+}$is right continuous and $\rho^{-}$is left continuous in $x$. According to the Lax-Oleinik Theorem [9], $\rho^{ \pm}$ coincides with the unique entropy solution to (2.9). Throughout the paper we will refer to (2.14) as the Lax-Oleinik formula.

Note that for each $t_{1}, t_{2}, y^{+}\left(x ; t_{1}, t_{2}\right)=y^{-}\left(x ; t_{1}, t_{2}\right)$ for all but at most countable $x$. At such point $\rho\left(x, t_{2}\right)$ is defined to be $\rho^{+}\left(x, t_{2}\right)=\rho^{-}\left(x, t_{2}\right)$. Moreover since $\rho$ is bounded, (2.14) implies

$$
\lim _{x \rightarrow \pm \infty} y^{+}\left(x ; t_{1}, t_{2}\right)=\lim _{x \rightarrow \pm \infty} y^{-}\left(x ; t_{1}, t_{2}\right)= \pm \infty \text {. }
$$

We denote the inverse of $y^{ \pm}\left(x ; t_{1}, t_{2}\right)$ in $x$-variable by $x^{ \pm}\left(y ; t_{1}, t_{2}\right)$, and they enjoy the following relationships

$$
\begin{aligned}
& y^{-}\left(x ; t_{1}, t_{2}\right)<y^{+}\left(x ; t_{1}, t_{2}\right) \quad \text { implies } x^{-}\left(y ; t_{1}, t_{2}\right)=x^{+}\left(y ; t_{1}, t_{2}\right)=x \\
& \quad \text { for } y \in\left(y^{-}\left(x ; t_{1}, t_{2}\right), y^{+}\left(x ; t_{1}, t_{2}\right)\right) \\
& x^{-}\left(y ; t_{1}, t_{2}\right)<x^{+}\left(y ; t_{1}, t_{2}\right) \text { implies } y^{-}\left(x ; t_{1}, t_{2}\right)=y^{+}\left(x ; t_{1}, t_{2}\right)=y \\
& \quad \text { for } x \in\left(x^{-}\left(y ; t_{1}, t_{2}\right), x^{+}\left(y ; t_{1}, t_{2}\right)\right) .
\end{aligned}
$$

By (2.13),

(2.16) $w\left(x, t_{2}\right)=\left(t_{2}-t_{1}\right) L\left(\frac{x-y^{ \pm}\left(x ; t_{2}, t_{1}\right)}{t_{2}-t_{1}}\right)+w\left(y^{ \pm}\left(x ; t_{2}, t_{1}\right), t_{1}\right)$.

This and the continuity of $w$ imply that $y^{+}\left(y^{-}\right)$is right (left) continuous in $x$. Indeed we can regard $y^{+}$and $y^{-}$as the right and left limit of some nondecreasing function.

Notation 2.4. - (i) $y^{ \pm}(x, t):=y^{ \pm}(x ; 0, t), \quad x^{ \pm}(y, t):=x^{ \pm}(y ; 0, t)$. If $y^{+}\left(x ; t_{1}, t_{2}\right)=y^{-}\left(x ; t_{1}, t_{2}\right)$, we denote it by $y\left(x ; t_{1}, t_{2}\right)$. Similarly we define $x\left(y ; t_{1}, t_{2}\right), x(y, t)$ and $y(x, t)$.

Remark 2.5. - It is worth mentioning that since $h$ is smooth, $L$ is strictly convex and $G$ is strictly increasing. 
Definition 2.6. - An absolutely continuous function $x:[0, T] \rightarrow \mathbb{R}$ is a Filippov solution to

$$
\left\{\begin{array}{l}
\frac{d x}{d t}=\gamma h^{\prime}(\rho(x, t)) \quad t>0 \\
x(0)=a
\end{array}\right.
$$

if for almost all $t \in[0, T]$,

$$
\frac{d x}{d t} \in\left[\gamma h^{\prime}\left(\rho^{+}(x(t), t)\right), \gamma h^{\prime}\left(\rho^{-}(x(t), t)\right)\right] .
$$

\section{Part 3}

Our first theorem settles the uniqueness problem for the initial value problem (2.17).

THEOREM 2.7. - Suppose

$$
\liminf _{\varepsilon \downarrow 0} \frac{1}{\varepsilon} \int_{a}^{a+\varepsilon} \rho_{0}(y) d y \leq \limsup _{\varepsilon \downarrow 0} \frac{1}{\varepsilon} \int_{a-\varepsilon}^{a} \rho_{0}(y) d y .
$$

Then there is at most one Filippov solution to (2.17).

The proof of this theorem will be given in $\S 3$.

We will show that $\frac{1}{L} x_{t L}$ converges to the unique Filippov solution of (2.17) providing $x_{0}=[a L]$.

Let $Q_{L}$ denote the law of the process $\left(\eta_{t}, x_{t}\right)$ where $x_{0}=[a L]$ and $\eta_{0}$ is distributed according to $\mu_{L}$ with $\mu_{L} \sim \rho_{0}$.

THEOREM 2.8. - In the case of SEP, suppose

$$
\int_{a}^{a+\delta}\left(1-\rho_{0}(y)\right) d y \int_{a-\delta}^{a} \rho_{0}(y) d y \neq 0
$$

for every positive $\delta$, and (2.18) holds. Then the law of $\left\{\frac{1}{L} x_{t L}\right\}$ is tight with respect to $Q_{L}$ and

$$
\lim _{L \rightarrow \infty} \int\left|\frac{1}{L} x_{t L}-x(a, t)\right| Q_{L}(d x)=0
$$

where $x(a, \cdot)$ denotes the unique solution of (2.17).

Note that we are requiring the initial density to be nonzero on the left of $a$ and less than one on the right of $a$, on a set of positive Lebsegue measure. 
We can also establish (2.20) for ZRP under the concavity assumption on the jump rate $g$.

Definition 2.9. - We say $g: \mathbb{N} \rightarrow \mathbb{R}$ is concave if the function $a(n):=g(n+1)-g(n)$ is nonincreasing in $n$.

THEOREM 2.10. - (2.20) holds for ZRP if $g$ is concave, $h^{\prime \prime}(\rho) \neq 0$, $p(1)=1$,

$$
\int_{a}^{a+\delta} \rho_{0}(y) d y \neq 0
$$

for every $\delta>0$, and (2.18) holds.

The condition $p(1)=1$ means that particles can jump to the right only. If we assume $p(-1)=1$, then (2.20) is replaced with $\int_{a-\delta}^{a} \rho_{0}(y) d y \neq 0$.

The proof of Theorem 2.8 and 2.10 will be given in $\$ 4$.

We can also prove (2.20) for processus misanthrope under some stringent assumptions on $b$. These assumptions will be stated at the end of $\S 4$.

To define the location of a second class particle, we take two configurations $\left(\eta_{0}, \zeta_{0}\right)$ that initially are different at a single site; $\zeta_{0}$ has an additional particle. At later times the discrepancy occurs at a single site we defined to be the location of the second class particle.

We imitate the above procedure macroscopically in the following manner. We take two initial densities $\rho_{0}$ and $\rho_{0, \delta}$ that are different only in the interval $[a-\delta, a+\delta]$. We consider the set of points $(x, t)$ at which $\rho(x, t) \neq \rho^{\delta}(x, t)$ where $\rho$ and $\rho^{\delta}$ solve (2.9) with initial densities $\rho_{0}$ and $\rho_{0, \delta}$ respectively. The set of discrepancies converges to a one-dimensional set that we expect to be the macroscopic trajectory of the second class particle.

We now briefly describe our strategy for the proof of Theorems 2.8 and 2.10 .

First we show that asymptotically the trajectory of the second class particle lies in the set of points $(x, t)$ at which $\rho^{\delta}(x, t) \neq \rho(x, t)$ for a suitably defined $\rho_{0, \delta}$. This will be done in $\S 4$.

Second, we prove that the set of points $(x, t)$ such that for every $\delta$, $\rho^{\delta}(x, t) \neq \rho(x, t)$ can be embedded in a neighborhood of the set

$$
\left\{(x, t): y^{-}(x, t) \leq a \leq y^{+}(x, t)\right\} .
$$

Finally we show that this set coincides with the trajectory of the unique Filippov solution of (2.17). This will be done in $\S 3$.

Our next theorem asserts that a disturbance in the initial density at point $a$ will be propagated along $x(a, t)$. 
THEOREM 2.11. - (a) Let $\left\{\rho_{0, \delta}\right\} \subseteq L^{1}$ be a uniformly bounded sequence of initial densities such that

$$
\rho_{0, \delta}(x)=\rho_{0}(x)
$$

for almost all $x \notin[a-\delta, a+\delta]$. Let $\rho_{\delta}^{ \pm}(x, t)$ denote the unique entropy solution given by the Lax-Oleinik formula and with initial density $\rho_{0, \delta}$. Then the set

$$
\begin{gathered}
\bigcap_{\delta>0} c \ell\left\{(x, t): 0<t \leq T \text { and either } \rho_{\delta}^{-}(x, t) \neq \rho^{-}(x, t)\right. \\
\text { or } \left.\rho_{\delta}^{+}(x, t) \neq \rho^{+}(x, t)\right\}
\end{gathered}
$$

is a subset of

$$
\left\{(x, t): 0 \leq t \leq T, x^{-}(a, t) \leq x \leq x^{+}(a, t)\right\} .
$$

Here "cl" denotes the topological closure.

(b) If (2.18) holds then

$$
x^{-}(a, t)=x^{+}(a, t)
$$

for all $t>0$.

Our next theorem identifies $x(a, t)$ to be the generalized characteristic emanating from point $a$.

Theorem 2.12. - Suppose (2.18) holds. Then $x(t)=x(a, t)$ is the unique Filippov solution to (2.17).

See the next section for a proof.

\section{PROPAGATION OF DISTURBANCES AND GENERALIZED CHARACTERISTICS}

This section is devoted to the proof of Theorems $2.7,2.11$ and 2.12 . We start with some preliminary lemmas.

Lemma 3.1. - Suppose $0 \leq t_{1}<t_{2}<t_{3}, y_{2} \in I\left(x ; t_{2}, t_{3}\right)$ and $y_{1} \in I\left(y_{2} ; t_{1}, t_{2}\right)$. Then

$$
\begin{aligned}
& \text { (i) } y_{1} \in I\left(x ; t_{1}, t_{3}\right) \\
& \text { (ii) } \frac{x-y_{2}}{t_{3}-t_{2}}=\frac{y_{2}-y_{1}}{t_{2}-t_{1}}=\frac{x-y_{1}}{t_{3}-t_{1}}
\end{aligned}
$$


Proof. - Since $y_{2} \in I\left(x ; t_{2}, t_{3}\right)$ and $y_{1} \in I\left(y_{2} ; t_{1}, t_{2}\right)$, we have

$$
\begin{gathered}
w\left(x, t_{3}\right)=\left(t_{3}-t_{2}\right) L\left(\frac{x-y_{2}}{t_{3}-t_{2}}\right)+w\left(y_{2}, t_{2}\right) \\
w\left(y_{2}, t_{2}\right)=\left(t_{2}-t_{1}\right) L\left(\frac{y_{2}-y_{1}}{t_{2}-t_{1}}\right)+w\left(y_{1}, t_{1}\right) .
\end{gathered}
$$

Therefore

$$
\begin{aligned}
w\left(x, t_{3}\right) & =\left(t_{3}-t_{2}\right) L\left(\frac{x-y_{2}}{t_{3}-t_{2}}\right) \\
& +\left(t_{2}-t_{1}\right) L\left(\frac{y_{2}-y_{1}}{t_{2}-t_{1}}\right)+w\left(y_{1}, t_{1}\right) \\
& \geq\left(t_{3}-t_{1}\right) L\left(\frac{x-y_{1}}{t_{3}-t_{1}}\right)+w\left(y_{1}, t_{1}\right)
\end{aligned}
$$

because $L$ is convex.

On the other hand, by (2.13),

$$
w\left(x, t_{3}\right)=\min _{y}\left\{\left(t_{3}-t_{1}\right) L\left(\frac{x-y}{t_{3}-t_{1}}\right)+w\left(y, t_{1}\right)\right\} .
$$

Therefore we have equality in (3.2) and this in turn implies (3.1). For (3.1)(ii) we have used the strict convexity of $L$.

Lemma 3.2. - Suppose $x^{-}(a, t)<x^{+}(a, t)$. Then

(i) $\limsup _{\varepsilon \downarrow 0} \frac{1}{\varepsilon} \int_{a-\varepsilon}^{a} \rho_{0}(y) d y<\liminf _{\varepsilon \downarrow 0} \frac{1}{\varepsilon} \int_{a}^{a+\varepsilon} \rho_{0}(y) d y$

(ii) For every $(x, s)$ with $s \in(0, t]$ and

$$
\frac{x^{-}(a, t)-a}{t}<\frac{x-a}{s}<\frac{x^{+}(a, t)-a}{t}
$$

we have $\rho(x, t)=G\left(\frac{x-a}{t}\right)$.

Proof. - If $x^{-}(a, t)<x^{+}(a, t)$, then $y(z, t)=a$ for every $z$ such that $x^{-}(a, t)<z<x^{+}(a, t)$. On the other hand, since $a$ is a minimizer of $t L\left(\frac{z-y}{t}\right)+w_{0}(y)$ for such $z$ we have

$$
\begin{aligned}
-G\left(\frac{z-a}{t}\right) & +\limsup _{\varepsilon \downarrow 0} \frac{w_{0}(a)-w_{0}(a-\varepsilon)}{\varepsilon} \leq 0 \leq-G\left(\frac{z-a}{t}\right) \\
& +\liminf _{\varepsilon \downarrow 0} \frac{w_{0}(a+\varepsilon)-w_{0}(a)}{\varepsilon}
\end{aligned}
$$


or

$$
\limsup _{\varepsilon \downarrow 0} \frac{1}{\varepsilon} \int_{a-\varepsilon}^{a} \rho_{0}(y) d y \leq G\left(\frac{z-a}{t}\right) \leq \liminf _{\varepsilon \downarrow 0} \frac{1}{\varepsilon} \int_{a}^{a+\varepsilon} \rho_{0}(y) d y .
$$

Since $G$ is strictly increasing, we cannot have equality. Therefore (i) holds. To prove (ii), we first observe that $y(z, t)=a$ implies $\rho(z, t)=$ $G\left(\frac{z-a}{t}\right)$. By continuity, we also have $y^{+}(z ; s, t)=y^{-}(z ; s, t)$ for every $s \in(0, t)$. We denote the common value by $x$. If $u \in I(x ; 0, s)$ then by (3.1) the points $(z, t),(x, s),(u, 0)$ are collinear and $u$ is in the set $I(z ; 0, t)=\{a\}$. Therefore $u=a$ and as a result $I(x ; 0, s)=\{a\}$ which in turn implies $\rho$ is continuous at $(x, s)$ with $\rho(x, t)=G\left(\frac{x-a}{s}\right)$. Since every point $(x, s)$ as in (ii) lies on a line segment connecting $(a, 0)$ to $(z, t)$ for some $z \in\left(x^{-}(a, t), x^{+}(a, t)\right)$, we are done.

COROLlaRY 3.3. - (i) If (2.18) holds, then $x^{-}(a, t)=x^{+}(a, t)$ for every positive $t$.

(ii) We have $x(a ; s, t):=x^{-}(a ; s, t)=x^{+}(a ; s, t)$ whenever $0<s<t$.

Proof. - Clearly (i) follows from Lemma 3.2(i). For (ii) first we observe that by Lax-Oleinik formula, $\rho^{-}(x, s) \geq \rho^{+}(x, s)$ if $s>0$. Therefore (2.18) holds for $s>0$, which implies (ii).

LEMMA 3.4. - For every $x$ and positive $t_{3}$, define

$$
\omega^{ \pm}\left(t_{2}\right)=y^{ \pm}\left(x ; t_{2}, t_{3}\right), \quad t_{2} \in\left[0, t_{3}\right) .
$$

Then

$$
\omega^{ \pm}\left(t_{2}\right)=\frac{t_{3}-t_{2}}{t_{3}} y^{ \pm}\left(x ; 0, t_{3}\right)+\frac{t_{2}}{t_{3}} x .
$$

Moreover if $t_{2} \in\left(0, t_{3}\right)$, then $\rho$ is continuous at $\left(\omega^{ \pm}\left(t_{2}\right), t_{2}\right)$.

Proof. - Let $y_{1}^{ \pm}=y^{ \pm}\left(y_{2} ; 0, t_{2}\right)$ where $y_{2}=y^{+}\left(x ; t_{2}, t_{3}\right)$. Then by (3.1) the points $\left(x, t_{3}\right), \quad\left(y_{2}, t_{2}\right),\left(y_{1}^{ \pm}, 0\right)$ are collinear. Thus $y_{1}^{+}=y_{1}^{-}$which implies the continuity of $\rho$ at $\left(y_{2}, t_{2}\right)=\left(\omega^{+}\left(t_{2}\right), t_{2}\right)$. Similarly one can prove the continuity of $\rho$ at $\left(\omega^{-}\left(t_{2}\right), t_{2}\right)$.

The linearity of $\omega^{ \pm}$follows from the fact that the left-hand side of

$$
\rho^{ \pm}\left(x, t_{3}\right)=G\left(\frac{x-y^{ \pm}\left(x ; t_{2}, t_{3}\right)}{t_{3}-t_{2}}\right)
$$

is independent of $t_{2}$. 
The next lemma establishes the semigroup property of $x^{ \pm}\left(y ; t_{1}, t_{2}\right)$ and $y^{ \pm}\left(x ; t_{1}, t_{2}\right)$.

Lemma 3.5. - Suppose $0 \leq t_{1}<t_{2}<t_{3}$. Then

$$
\begin{aligned}
& x^{ \pm}\left(a ; t_{1}, t_{3}\right)=x\left(x^{ \pm}\left(a ; t_{1}, t_{2}\right) ; t_{2}, t_{3}\right) \\
& y^{ \pm}\left(x ; t_{1}, t_{3}\right)=y\left(y^{ \pm}\left(x ; t_{2}, t_{3}\right) ; t_{1}, t_{3}\right)
\end{aligned}
$$

Proof. - Step 1. - Choose $y_{2} \in I\left(x ; t_{2}, t_{3}\right)$ and $y_{1} \in I\left(y_{2} ; t_{1}, t_{2}\right)$. By (3.1), the points $\left(x, t_{3}\right),\left(y_{2}, t_{2}\right),\left(y_{1}, t_{1}\right)$ are collinear and

$$
\alpha, \beta \in I\left(x ; t_{1}, t_{3}\right)
$$

where

$$
\begin{aligned}
& \alpha=y^{-}\left(y^{-}\left(x ; t_{2}, t_{3}\right) ; t_{1}, t_{2}\right) \\
& \beta=y^{+}\left(y^{+}\left(x ; t_{2}, t_{3}\right) ; t_{1}, t_{2}\right)
\end{aligned}
$$

To prove (3.8) it suffices to choose $y_{2}=y^{ \pm}\left(x ; t_{2}, t_{3}\right)$ and $y_{1}=$ $y^{ \pm}\left(y_{2} ; t_{1}, t_{2}\right)$. Equivalently (3.7) can be written as

$$
y^{-}\left(x ; t_{1}, t_{3}\right) \leq \alpha \leq \beta \leq y^{+}\left(x ; t_{1}, t_{3}\right) .
$$

Step 2. - To prove (3.5), let $z^{ \pm}=x^{ \pm}\left(a ; t_{1}, t_{2}\right)$ and $x^{ \pm}=x\left(z^{ \pm} ; t_{2}, t_{3}\right)$ which is well defined by Corollary 3.3(ii) since $t_{2}>0$. Then by definition

$$
\begin{aligned}
& y^{-}\left(x^{ \pm} ; t_{2}, t_{3}\right) \leq z^{ \pm} \leq y^{+}\left(x^{ \pm} ; t_{2}, t_{3}\right) \\
& y^{-}\left(y^{-}\left(x^{ \pm} ; t_{2}, t_{3}\right) ; t_{1}, t_{2}\right) \leq a \leq y^{+}\left(y^{+}\left(x^{ \pm} ; t_{2}, t_{3}\right) ; t_{1}, t_{2}\right) .
\end{aligned}
$$

Now (3.9) can be used to conclude

$$
y^{-}\left(x^{ \pm} ; t_{1}, t_{3}\right) \leq a \leq y^{\dagger}\left(x^{ \pm} ; t_{1}, t_{3}\right)
$$

that in turn implies

$$
x^{-}\left(a ; t_{1}, t_{3}\right) \leq x^{-} \leq x^{+} \leq x^{+}\left(a ; t_{1}, t_{3}\right) .
$$

If $x^{-}\left(a ; t_{1}, t_{3}\right)=x^{+}\left(a ; t_{1}, t_{3}\right)$, then $x^{ \pm}\left(a ; t_{1}, t_{3}\right)=x^{ \pm}$which implies (3.5).

Step 3. - To complete the proof of (3.5), it is left to treat the case $x^{-}\left(a ; t_{1}, t_{3}\right)<x^{+}\left(a ; t_{1}, t_{3}\right)$. Note that by Corollary 3.3(ii), $t_{1}$ can only be zero. 
Define $v^{ \pm}$by

$$
\frac{v^{ \pm}-a}{t_{2}}=\frac{x^{ \pm}\left(a ; 0, t_{3}\right)-a}{t_{3}} .
$$

By Lemma 3.2, $\rho(z, t)=G\left(\frac{z-a}{t_{2}}\right)$ for every $z \in\left(v^{-}, v^{+}\right)$. In particular $\rho$ is continuous at such $z$ which implies $y\left(z ; 0, t_{2}\right)=a$. Therefore

$$
z^{-} \leq v^{-} \leq v^{+} \leq z^{+}
$$

Our goal is to show $x^{ \pm}\left(a ; 0, t_{3}\right)=x^{ \pm}$and by (3.10) it suffices to rule out $x^{+}<x^{+}\left(a ; 0, t_{3}\right)$ and $x^{-}>x^{-}\left(a ; 0, t_{3}\right)$. We only treat the former one because the latter one can be treated in the same way.

If $x^{+}<x^{+}\left(a ; 0, t_{3}\right)$, then by Lemma 3.2(ii), $\rho$ is continuous at $\left(x^{+}, t_{3}\right)$. Therefore $y\left(x^{+} ; t_{2}, t_{3}\right)=z^{+}$and by (3.1) the points $\left(x^{+}, t_{3}\right),\left(z^{+}, t_{2}\right),(a, 0)$ are collinear. This implies $z^{+}<v^{+}$which contradicts (3.11).

Final step. - Let $\alpha^{*}=y^{-}\left(x ; t_{1}, t_{3}\right)$. By (3.9) we already know $\alpha^{*} \leq \alpha$. To prove (3.6) we need to show $\alpha \leq \alpha^{*}$.

By definition we have $x \in\left[p^{-}, p^{+}\right]$where

$$
p^{ \pm}=x^{ \pm}\left(\alpha^{*} ; t_{1}, t_{3}\right)=x\left(x^{ \pm}\left(\alpha^{*} ; t_{1}, t_{2}\right) ; t_{2}, t_{3}\right)
$$

where, for the second equality we have used (3.5). Therefore

$$
y^{-}\left(p^{ \pm} ; t_{2}, t_{3}\right) \leq x^{ \pm}\left(\alpha^{*} ; t_{1}, t_{2}\right) \leq y^{+}\left(p^{ \pm} ; t_{2}, t_{3}\right)
$$

which in turn implies

$$
y^{-}\left(y^{-}\left(p^{+} ; t_{2}, t_{3}\right) ; t_{1}, t_{2}\right) \leq \alpha^{*}
$$

Since $x \leq p^{+}$, we conclude $\alpha \leq \alpha^{*}$. Similarly we can show $\beta=$ $y^{+}\left(x ; t_{1}, t_{3}\right)$.

In our first application of the semigroup property we show that indeed $w^{ \pm}$in Lemma 3.4 are the classical characteristics.

LemMa 3.6. - Let $w^{ \pm}$be defined as in Lemma 3.4. Then for every $t_{2} \in\left(0, t_{3}\right)$, we have $\rho\left(w^{ \pm}\left(t_{2}\right), t_{2}\right)=\rho^{ \pm}\left(x, t_{3}\right)$.

Proof. - By semigroup property (3.6),

$$
y\left(w^{ \pm}\left(t_{2}\right) ; 0, t_{2}\right)=y^{ \pm}\left(x ; 0, t_{3}\right)=w^{ \pm}(0) .
$$

Vol. $12, n^{\circ} 2-1995$. 
(Here we are using the continuity of $\rho$ at $\left(w^{ \pm}\left(t_{2}\right),\left(t_{2}\right)\right)$. Hence

$$
\begin{aligned}
\rho\left(w^{ \pm}\left(t_{2}\right), t_{2}\right) & =G\left(\frac{w^{ \pm}\left(t_{2}\right)-w^{ \pm}(0)}{t_{2}}\right) \\
& =G\left(\frac{x-w^{ \pm}(0)}{t_{3}}\right) \\
& =\rho^{ \pm}\left(x, t_{3}\right) .
\end{aligned}
$$

Here we used (3.4) for the second equality.

The semigroup property (3.5) can be used to show that in fact $x^{t}(a ; 0, t)$ are Filippov solutions to (2.17).

LEMmA 3.7. - Let $x(t)=x^{ \pm}(a ; 0, t)$. Then $x$ is Lipschitz and a Filippov solution to the initial value problem (2.17).

Proof. - Let $A=\left\{t: \rho^{+}(x(t), t)=\rho^{-}(x(t), t)\right\}$. By the RankineHugoniot formula, for almost all $t \notin A$ we have

$$
\frac{d x}{d t}(t)=\gamma \frac{h\left(\rho^{+}(x(t), t)\right)-h\left(\rho^{-}(x(t), t)\right)}{\rho^{+}(x(t), t)-\rho^{-}(x(t), t)}
$$

(see Dafermos [2], Theorem 3.1). Therefore for almost all $t \notin A,(2.17 a)$ holds.

Fix a positive $t_{0} \in A$ and let $t \in A \cap\left(t_{0},+\infty\right)$. Since $t_{0}, t \in A$, we have

$$
\left\{\begin{array}{l}
\rho^{+}\left(x\left(t_{0}\right), t_{0}\right)=\rho^{-}\left(x\left(t_{0}\right), t_{0}\right) \\
\rho^{+}(x(t), t)=\rho^{-}(x(t), t) .
\end{array}\right.
$$

Therefore

$$
y^{+}\left(x(t) ; t_{0}, t\right)=y^{-}\left(x(t) ; t_{0}, t\right) .
$$

By semigroup property

$$
x(t)=x\left(x\left(t_{0}\right) ; t_{0}, t\right)
$$

which implies

$$
x\left(t_{0}\right)=y\left(x(t) ; t_{0}, t\right) .
$$

By (3.13), $w\left(y, t_{0}\right)$ is differentiable at $y=x\left(t_{0}\right)$. On the other hand, at $y\left(x(t) ; t_{0}, t\right)$ the minimum of

$$
\left(t-t_{0}\right) L\left(\frac{x(t)-y}{t-t_{0}}\right)+w\left(y, t_{0}\right)
$$


is attained. Hence (3.14) implies

$$
G\left(\frac{x(t)-x\left(t_{0}\right)}{t-t_{0}}\right)=\frac{\partial w}{\partial y}\left(x\left(t_{0}\right), t_{0}\right)=\rho\left(x\left(t_{0}\right), t_{0}\right) .
$$

By the Lax-Oleinik formula we also have

$$
G\left(\frac{x(t)-x\left(t_{0}\right)}{t-t_{0}}\right)=\rho(x(t), t) .
$$

Therefore

$$
\frac{x(t)-x\left(t_{0}\right)}{t-t_{0}}=\gamma h^{\prime}(\rho(x(t), t))=\gamma h^{\prime}\left(\rho\left(x\left(t_{0}\right), t_{0}\right)\right)
$$

for every $t \in A \cap\left(t_{0},+\infty\right)$.

From this we conclude that for almost all $t_{0} \in A$,

$$
\frac{d x}{d t}\left(t_{0}\right)=\gamma h^{\prime}\left(\rho\left(x\left(t_{0}\right), t_{0}\right)\right)
$$

Finally we show $x(t)$ is Lipschitz. By semigroup property, it suffices to show that there exists a constant $C$ such that for every $z \in \mathbb{R}$,

$$
\left|x^{ \pm}(z ; t, t+\delta)-z\right| \leq C \delta
$$

Let $x=x^{ \pm}(z ; t, t+\delta)$. Then

$$
y^{-}(x ; t, t+\delta) \leq z \leq y^{+}(x ; t, t+\delta) .
$$

Therefore we only need to show

$$
\left|y^{ \pm}(x ; t, t+\delta)-x\right| \leq C \delta
$$

By the Lax-Oleinik formula (2.14),

$$
x-y^{ \pm}(x ; t, t+\delta)=\delta \gamma h^{\prime}\left(\rho^{ \pm}(x, t+\delta)\right)
$$

which implies (3.15) because $\rho$ is bounded.

It has been shown in Dafermos [2] that the initial value problem

$$
\left\{\begin{array}{l}
\frac{d z}{d t}=\gamma h^{\prime}(\rho(z, t)), \quad t>t_{0} \\
z\left(t_{0}\right)=z_{0}
\end{array}\right.
$$

has a unique solution providing $t_{0}>0$. By Lemma 3.7 this solution is $z(t)=x\left(z_{0} ; t_{0}, t\right)$. If $(2.18)$ holds, we have a unique solution even when $t_{0}=0$. In fact we can prove more: 
Theorem 3.8. - Let $z^{ \pm}(t)=x^{ \pm}(a ; 0, t)$. Then $z^{+}, z^{-}$solve (2.17). Moreover, for every solution $z(t)$ to $(2.17)$ we have

$$
z^{-}(t) \leq z(t) \leq z^{+}(t)
$$

for all $t \geq 0$.

Proof. - Let $z(t)$ be any solution to (2.17) and suppose to the contrary (3.17) does not hold. Suppose $z\left(t_{1}\right) \notin\left[z^{-}\left(t_{1}\right), z^{+}\left(t_{1}\right)\right]$ for some positive $t_{1}$. Since $z^{+}, z^{-}$both solve (3.16) and we have uniqueness for (3.16) if $t_{0}>0$, we conclude that in fact $z(t) \notin\left[z^{-}(t), z^{+}(t)\right]$ for every $t \in\left(0, t_{1}\right]$. Suppose for example $z(t)<z^{-}(t)$ for every $t \in\left(0, t_{1}\right]$. The other case can be treated analogously.

Since (3.16) has a unique solution if $t_{0}>0$, we have

$$
z(t)=x\left(z\left(t_{0}\right) ; t_{0}, t\right)
$$

for every positive $t_{0}$. This in turn implies

$$
y^{-}\left(z(t) ; t_{0}, t\right) \leq z\left(t_{0}\right) \leq y^{+}\left(z(t) ; t_{0}, t\right) \text {. }
$$

By Lemma 3.4, the points $\left(y^{ \pm}\left(z(t) ; t_{0}, t\right), t_{0}\right)$ are collinear if we vary $t_{0}$ and fix $t$. By passing to the limit $t_{0} \downarrow 0$, we obtain

$$
y^{-}(z(t) ; 0, t) \leq a \leq y^{+}(z(t) ; 0, t)
$$

If

$$
y^{-}(z(t) ; 0, t) \leq a<y^{+}(z(t) ; 0, t)
$$

then for sufficiently small $s$,

$$
z^{-}(s)<y^{+}(z(t) ; s, t)
$$

because by Lemma 3.4, $y^{+}(z(t) ; s, t)$ is continuous (in fact linear) in $s$ and $z^{-}(0)=a$. But this implies

$$
z^{-}(t)=x\left(z^{-}(s) ; s, t\right) \leq z(t)
$$

which contradicts our assumption $z^{-}(t)>z(t)$. Hence the strict inequality in (3.18) cannot be true;

$$
y^{+}(z(t) ; 0, t)=a
$$


This in turn implies

$$
x^{-}(a ; 0, t) \leq z(t)
$$

which is not possible. Thus $z(t) \in\left[z^{-}(t), z^{+}(t)\right]$.

An immediate corollary to Theorem 3.8 is Theorem 2.12. The following example shows that without $(2.18)$ we may have more than one solution to $(2.17)$.

$$
\begin{aligned}
\text { Example. - Let } \gamma h(\rho)=\frac{1}{2} \rho^{2} \text { and } \\
\rho(x, t)=\left\{\begin{array}{cc}
0 & x \leq 0 \\
\frac{x}{t} & 0<x<t \\
1 & x \geq t .
\end{array}\right.
\end{aligned}
$$

Then $x(t)=c t$ is a solution to $(2.17)$ for every $c \in[0,1]$. One can easily check that $z^{-}(t) \equiv 0$ and $z^{+}(t)=t$.

The rest of this section is devoted to the proof of Theorem 2.11.

First we start with a lemma that asserts $y^{ \pm}$are nonincreasing with respect to the initial condition $\rho_{0}$.

Lemma 3.9. - Suppose $\rho_{0}, \hat{\rho}_{0} \in L^{1} \cap L^{\infty}$ and

$$
\rho_{0} \leq \hat{\rho}_{0}
$$

almost everywhere. Let $y^{ \pm}(x, t), \hat{y}^{ \pm}(x, t), \rho^{ \pm}(x, t), \hat{\rho}^{ \pm}(x, t)$ denote the corresponding minimizer and entropy solutions given by the Lax-Oleinik formula. Then for every $t>0$,

$$
y^{ \pm}(x, t) \geq \hat{y}^{ \pm}(x, t)
$$

and

$$
\rho^{ \pm}(x, t) \leq \hat{\rho}^{ \pm}(x, t)
$$

Proof. - By the Lax-Oleinik formula, (3.19) implies (3.20).

Set

$$
\psi(y)=t L\left(\frac{x-y}{t}\right)+w_{0}(y)
$$


and let $I(x, t)$ denote the set of points $y$ at which the minimum of $\psi$ is attained. Analogously we define $\widehat{w}_{0}, \widehat{\psi}$ and $\hat{I}(x, t)$. Suppose $z_{1} \in I(x, t)$ and $z_{2} \geq z_{1}$. Then

$$
\begin{aligned}
w_{0}\left(z_{2}\right)+t L\left(\frac{x-z_{2}}{t}\right) & \geq w_{0}\left(z_{1}\right)+t L\left(\frac{x-z_{1}}{t}\right) \\
w_{0}\left(z_{1}\right)-w_{0}\left(z_{2}\right) & \geq \widehat{w}_{0}\left(z_{1}\right)-\widehat{w}_{0}\left(z_{2}\right) .
\end{aligned}
$$

Therefore,

$$
\widehat{w}_{0}\left(z_{2}\right)+t L\left(\frac{x-z_{2}}{t}\right) \geq \widehat{w}_{0}\left(z_{1}\right)+t L\left(\frac{x-z_{1}}{t}\right) .
$$

Hence either $z_{1} \in \hat{I}(x, t)$ or $\hat{I}(x, t) \subseteq\left(-\infty, z_{1}\right)$. Similarly if $y_{1} \in \hat{I}(x, t)$ then either $y_{1} \in I(x, t)$ or $I(x, t) \subseteq\left(y_{1},+\infty\right)$. Now (3.19) follows if we choose $z_{1}=y^{-}(x, t)$ and $y_{1}=\hat{y}^{+}(x, t)$.

Proof of Theorem 2.11. - (a) Step 1. - Since $\left\{\rho_{0, \delta}: \delta>0\right\}$ is uniformly bounded, there exists a constant $k$ such that

$$
\begin{aligned}
& q_{\delta}(x) \leq \rho_{0}(x) \leq p_{\delta}(x) \\
& q_{\delta}(x) \leq \rho_{0, \delta}(x) \leq p_{\delta}(x)
\end{aligned}
$$

where $q_{\delta}(x)=p_{\delta}(x)=\rho_{0}(x)$ for $x \notin[a-\delta, a+\delta]$, and

$$
p_{\delta}(x)=-q_{\delta}(x)=k
$$

for $x \in[a-\delta, a+\delta]$. Let $p_{\delta}^{ \pm}(x, t)$ and $q_{\delta}^{ \pm}(x, t)$ denote the corresponding entropy solutions with the initial densities $p_{\delta}(x)$ and $q_{\delta}(x)$ respectively.

If at a point $(x, t)$ we have

$$
p_{\delta}^{ \pm}(x, t)=\rho^{ \pm}(x, t) \quad \text { and } \quad q_{\delta}^{ \pm}(x, t)=\rho^{ \pm}(x, t)
$$

then $\rho^{ \pm}(x, t)=\rho_{\delta}^{ \pm}(x, t)$ as well. Therefore the set (2.22) is a subset of the closure of the set of points $(x, t)$ at which, for every $\delta>0$, at least one of the four relations in (3.22) fails. Hence, one of these relations would fail for infinitely many $\delta$ which by monotonicity property (3.20) would fail for all sufficiently small $\delta$. Let $\delta_{0}>0$ and

$$
D^{ \pm}:=\bigcap_{0<\delta<\delta_{0}} D_{\delta}^{ \pm}
$$

where $D_{\delta}^{ \pm}=c \ell F_{\delta}^{ \pm}$and

$$
F_{\delta}^{ \pm}=\left\{(x, t): p_{\delta}^{ \pm}(x, t) \neq \rho^{ \pm}(x, t), 0<t<T\right\} .
$$


We will show that $D^{ \pm}$is a subset of (2.23) for every $\delta_{0}$. The other cases corresponding to $q_{\delta}^{ \pm}$can be treated analogously.

Step 2. - It suffices to show

$$
D^{+} \cup D^{-} \subseteq\left\{(x, t): y^{-}(x, t) \leq a \leq y^{+}(x, t), 0 \leq t \leq T\right\}
$$

This is equivalent to showing that if $a \notin\left[y^{-}(x, t), y^{+}(x, t)\right]$ then there exists a positive $\delta$ such that

$$
B_{\delta}(x, t) \cap\left(F_{\delta}^{+} \cup F_{\delta}^{-}\right)=\emptyset
$$

where $B_{\delta}(x, t)=\{(z, s):|z-x|<\delta,|s-t|<\delta\}$.

Pick a point $(x, t)$ with $t \in(0, T)$ and $a \notin\left[y^{-}(x, t), y^{+}(x, t)\right]$. For each $t, y(\cdot, t)$ is nondecreasing and $y^{+}, y^{-}$denote its right and left limits. Therefore for some $\delta_{0}>0$,

$$
a \notin\left[y^{-}\left(x-\delta_{0}, t\right), y^{+}\left(x+\delta_{0}, t\right)\right] \text {. }
$$

This means that either $a<y^{-}\left(x-\delta_{0}, t\right)$ or $a>y^{+}\left(x-\delta_{0}, t\right)$. By semigroup property (3.6) and estimate (3.15), $y^{ \pm}(x, t)$ is Lipschitz in $t$. Therefore we may choose a smaller $\delta_{0}$ to ensure that either

$$
a+\delta_{0}<y^{-}\left(x-\delta_{0}, s\right) \quad \text { for } \quad s \in\left[t-\delta_{0}, t+\delta_{0}\right]
$$

or

$$
a-\delta_{0}>y^{+}\left(x+\delta_{0}, s\right) \text { for } s \in\left[t-\delta_{0}, t+\delta_{0}\right]
$$

Step 3. - We would like to show that both (3.27) and (3.28) imply (3.25). We start with (3.27). Write

$$
\begin{gathered}
\psi_{\delta}(y, x, t)=t L\left(\frac{x-y}{t}\right)+w_{0, \delta}(y) \\
\psi(y, x, t)=t L\left(\frac{x-y}{t}\right)+w_{0}(y)
\end{gathered}
$$

where

$$
w_{0, \delta}(y)=\int_{-\infty}^{y} p_{\delta}(z) d z
$$

Let $I^{\delta}(x, t)$ denote the set of points $y$ at which the minimum of $\psi_{\delta}$ is attained. We write $y_{\delta}^{+}(x, t), y_{\delta}^{-}(x, t)$ for the largest and smallest values in $I^{\delta}(x, t)$ respectively. 
Since there exists a constant $k(\delta)$ such that $w_{0, \delta}(y)=w_{0}(y)+k(\delta)$ for $y \geq a+\delta$, any minimizer of $\psi_{\delta}$ in the interval of $[a+\delta,+\infty)$ is also a minimizer of $\psi$. Suppose (3.27) holds for $(x, t)$. Then for every $\delta \in\left(0, \delta_{0}\right]$, every $s \in[t-\delta, t+\delta]$ and $z \in[x-\delta, x+\delta]$, we have

$$
a+\delta \leq a+\delta_{0}<y^{-}\left(x-\delta_{0}, s\right) \leq y^{-}(z, s) .
$$

Hence if $y_{\delta}^{-}(z, s) \geq a+\delta$, then $\psi_{\delta}, \psi$ have the same set of minimizers which implies $y_{\delta}^{ \pm}(z, s)=y^{ \pm}(z, s)$ that in turn implies $p_{\delta}^{ \pm}(z, s)=\rho^{ \pm}(z, s)$ or $(z, s) \notin F_{\delta}^{+} \cup F_{\delta}^{-}$. Therefore if (3.25) fails for every $\delta$ then for every $\delta \in\left(0, \delta_{0}\right]$, there exists a pair $(z(\delta), s(\delta))$ such that

$$
\begin{aligned}
& s(\delta) \in[t-\delta, t+\delta], \\
& z(\delta) \in[x-\delta, x+\delta], \quad \text { and } \\
& y_{\delta}^{-}(z(\delta), s(\delta))<a+\delta .
\end{aligned}
$$

Since $\psi_{\delta}=\psi$ in the interval $(-\infty, a-\delta]$, it is not hard to show that the sequence $y_{\delta}^{-}(z(\delta), s(\delta))$ is bounded below. We also know

$$
\lim _{\delta \rightarrow 0} \sup _{x, y, t}\left|\psi_{\delta}(y, x, t)-\psi(y, x, t)\right|=0 .
$$

Hence

$$
\lim _{\delta \rightarrow 0} \psi_{\delta}\left(y_{\delta}^{-}(z(\delta), s(\delta)), z(\delta), s(\delta)\right)=\inf _{y} \psi(y, x, t) .
$$

If, for a subsequence we have

$$
\lim _{\delta \rightarrow 0} y_{\delta}^{-}(z(\delta), s(\delta))=b
$$

then

$$
\psi(b, x, t)=\inf _{y} \psi(y, x, t)
$$

which implies

$$
y^{-}(x, t) \leq b \leq y^{+}(x, t) .
$$

On the other hand, (3.30) implies $b \leq a$, so $y^{-}(x, t) \leq a$, contradicting (3.27). Thus (3.27) implies (3.25).

Final Step. - It is left to show that (3.28) implies (3.25). Suppose (3.28) holds. By monotonicity (3.19) we have

$$
\begin{aligned}
& a-\delta_{0}>y_{\delta}^{+}(z, s) \\
& a-\delta_{0}>y^{+}(z, s)
\end{aligned}
$$


for every $(z, s) \in\left[x-\delta_{0}, x+\delta_{0}\right] \times\left[t-\delta_{0}, t+\delta_{0}\right]$. This implies $y^{ \pm}(z, s)=y_{\delta_{0}}^{ \pm}(z, s)$ because $p_{\delta_{0}}(y)=w_{0}(y)$ for $y \in\left(-\infty, a-\delta_{0}\right]$ and as a result $\psi_{\delta_{0}}, \psi$ have the same set of minimizers. Thus $\rho^{ \pm}(z, s)=p_{\delta_{0}}^{ \pm}(z, s)$ which implies (3.25).

\section{SECOND CLASS PARTICLES}

In this section we prove Theorems 2.8 and 2.10 . The nearest neighbor jump assumption in the SEP case and the concavity of $g$ plus the uniform asymmetry in the case of ZRP imply that a single second class particle will be trapped between a bunch of second class particles. This can be utilized to reduce the law of large numbers for a single second class particle to the law of large numbers for the density of second class particles. We then apply Theorems 2.11 and 2.12 to complete the proof of Theorems 2.8 and 2.10.

We start with the proof of tightness.

LемMA 4.1. - The sequence of processes $\left\{\frac{1}{L} x_{t L}\right\}$ is tight.

Proof. - Recall that for every $f$, the following are martingale:

$$
\begin{aligned}
M_{t} & :=f\left(\eta_{t}, x_{t}\right)-f\left(\eta_{0}, x_{0}\right)-\int_{0}^{t} \mathcal{A} f\left(x_{s}, \eta_{s}\right) d s, \\
N_{t} & :=M_{t}^{2}-\int_{0}^{t}\left(\mathcal{A} f^{2}-2 f \mathcal{A} f\right)\left(x_{s}, \eta_{s}\right) d s .
\end{aligned}
$$

where $\mathcal{A}$ is defined by (2.11).

If we choose $f(\eta, x)=x$, we have

$$
\frac{1}{L} x_{t L}=\frac{1}{L} x_{0}+\frac{1}{L} \int_{0}^{t L} V\left(\eta_{s}, x_{s}\right) d s+\frac{1}{L} M_{t L}
$$

where

$$
\begin{aligned}
V(\eta, x) & =\sum_{k} p(k) k(b(\eta(x)+1, \eta(x+k)))-(b(\eta(x), \eta(x+k))) \\
& -\sum_{k} p(k) k(b(\eta(x-k), \eta(x))-b(\eta(x-k), \eta(x)+1)) .
\end{aligned}
$$

Since $V$ is bounded, the middle term in the right-hand side of (4.2) is uniformly Lipschitz in $t$. Therefore it suffices to show

$$
\lim _{L \rightarrow \infty} E\left[\sup _{0 \leq t \leq t_{0}} \frac{1}{L} M_{t L}\right]^{2}=0 .
$$


By Doob's inequality we only need to show $\lim _{L \rightarrow \infty} \frac{1}{L^{2}} E M_{t L}^{2}=0$. Using (4.1) again we have

$$
\begin{aligned}
\frac{1}{L^{2}} E M_{t L}^{2}= & \frac{1}{L^{2}} \int_{0}^{t L} \sum_{k} p(k) k^{2}\left(b\left(\eta_{s}(x)+1, \eta_{s}(x+k)\right)\right. \\
& +b\left(\eta_{s}(x-k), \eta_{s}(x)\right)-b\left(\eta_{s}(x), \eta_{s}(x+k)\right) \\
& \left.-b\left(\eta_{s}(x-k), \eta_{s}(x)+1\right)\right) d s
\end{aligned}
$$

that clearly goes to zero as $L \rightarrow \infty$.

Proof of Theorem 2.8. - The proof of (2.20) is carried out in several steps.

Step 1. - Write $q=p(1), p=p(-1)$ and assume $p \geq q$. Let $\left(\eta_{t}, x_{t}\right)$ be a process generated by $\mathcal{A}$ such that initially $\eta_{0}$ is distributed according to $\mu_{L}$ with $\mu_{L} \sim \rho_{0}$ and $x_{0}=[a L]$. In the case of SEP, $\mathcal{A}$ can be written as

$$
\begin{aligned}
\mathcal{A} f(\eta, x)= & \sum_{u, v \neq x} p(v-u) \eta(u)(1-\eta(v))\left(f\left(\eta^{u v}, x\right)-f(\eta, x)\right) \\
& +(p-q)(1-\eta(x-1))(f(\eta, x-1)-f(\eta, x)) \\
& +(p-q) \eta(x+1)\left(f\left(\eta^{x+1, x}, x+1\right)-f(\eta, x)\right) \\
& +q\left(f\left(\eta_{x, x+1}, x+1\right)+f\left(\eta_{x, x-1}, x-1\right)-2 f(\eta, x)\right)
\end{aligned}
$$

where $\eta^{u, v}$ is as before and

$$
\eta_{u, v}(z)= \begin{cases}\eta(v) & \text { if } z=u \\ \eta(u) & \text { if } z=v \\ \eta(z) & \text { otherwise }\end{cases}
$$

Here we are assuming that initially $\eta_{0}\left(x_{0}\right)=0$. This implies $\eta_{t}\left(x_{t}\right)=0$ for all times.

The middle terms in (4.3) represent the purely asymmetric part of the generator. The last term corresponds to the symmetric part of the generator. According to the last term, the second class particle jumps as a symmetric random walk and if there is an $\eta$-particle at the site it is jumping to, the $\eta$-particle and the second class particle exchange sites. Note that the rate of a jump in the last term does not depend on the confiuration. Here we used the new notation $\eta_{u, v}$ to simplify the notation and put emphasis on the symmetric feature of the last term. 
Step 2. - Let $\zeta_{t}$ be a process generated by $\mathcal{L}$ with initial distribution $\mu_{L}^{\delta} \sim \rho_{0, \delta}$ where

$$
\rho_{0, \delta}(x)= \begin{cases}1 & \text { if } x \in[a, a+\delta] \\ \rho_{0}(x) & \text { otherwise }\end{cases}
$$

By monotonicity we can write

$$
\zeta_{t}=\eta_{t}+\alpha_{t}, \quad \alpha_{t} \in\{0,1\}^{\mathbb{Z}} .
$$

We regard $\alpha_{t}(u)$ as the occupation number of second class particles. One can easily write down the generator of $(\eta, \alpha)$. For our purposes we will write the generator in an appropriate way.

As before we decompose the generator into symmetric and purely asymmetric parts. Let $\mathcal{B}$ denote the infinitesimal generator of the process $(\eta, \alpha)$. We write

$$
\begin{aligned}
\mathcal{B} f(\eta, \alpha) & =(p-q) \sum_{u} \eta(u)(1-\zeta(u-1))\left(f\left(\eta^{u, u-1}, \alpha\right)-f(\eta, \alpha)\right) \\
& +(p-q) \sum_{u}^{u} \alpha(u)(1-\zeta(u-1))\left(f\left(\eta, \alpha^{u, u-1}\right)-f(\eta, \alpha)\right) \\
& +(p-q) \sum_{u} \alpha(u) \eta(u+1)\left(f\left(\eta^{u+1, u}, \alpha^{u, u+1}\right)-f(\eta, \alpha)\right) \\
& +q \sum_{u}\left(f\left(\eta_{u, u+1}, \alpha_{u, u+1}\right)-f(\eta, \alpha)\right)
\end{aligned}
$$

The first three terms represent the purely asymmetric part of the generator. The last term corresponds to the symmetric part. According to this part, particles move as symmetric random walks. At the transition times, if the sites of a bond are occupied by an $\eta$-particle and an $\alpha$-particle, they exchange sites.

Step 3. - If $q=0$, it is possible to couple the processes $\eta, \alpha, x$ so that for all times there is no $\alpha$-particle on the left of $x_{t}$. This is not possible if $q \neq 0$. However the special form of the last term in (4.4) makes it possible to construct a coupling so that a good number of $\alpha$-particles stay on the right of $x_{t}$. The idea is as follows. We label $\alpha$-particles from 1 to $N$ where $N$ is the total number of $\alpha$-particles. We let a particle of a given label jump according to the following rules:

(1) with rate $p-q$ it jumps to the left if there is no $\zeta$-particle there,

(2) with rate $p-q$ it jumps to the right if there is no $\alpha$-particle there, but there is an $\eta$ particle, 
(3) with rate $q$ it jumps as a symmetric random walk.

Now it is possible to couple $x_{t}$ with the labeled $\alpha$-particles so that an $\alpha$-particle of a fixed label stays on the right of $x_{t}$ at all times. Of course it is possible that the other $\alpha$-particles cross $x_{t}$ and jump to its left. Since a particle of fixed label with an arbitrary initial location stays on the right of $x_{t}$, we conclude that a good number of $\alpha$-particles must be on the right of $x_{t}$ as well. To make this precise we start with the precise definition of the labeled particles and their dynamics.

Let $z_{1}, z_{2}, \ldots, z_{N}$ denote the locations of the $\alpha$-particles:

$$
\begin{aligned}
& \mathbf{z}=\left(z_{1} \ldots z_{N}\right), \\
& \alpha(u ; \mathbf{z}):=\alpha(u)= \begin{cases}1 & \text { if } u=z_{i} \text { for some } i \\
0 & \text { otherwise }\end{cases} \\
& \zeta(u ; \mathbf{z})=\eta(u)+\alpha(u ; \mathbf{z}) \\
& N=\sum_{u} \alpha(u) .
\end{aligned}
$$

The infinitesimal generator of $\left(\eta_{t}, \mathbf{z}_{t}\right)$ is as follows:

$$
\text { (4.5) } \begin{aligned}
\widehat{\mathcal{B}} & f(\eta, \mathbf{z})= \\
= & (p-q) \sum_{u} \eta(u)(\mathbf{1}-\zeta(u-1 ; \mathbf{z}))\left(f\left(\eta^{u, u-1}, \mathbf{z}\right)-f(\eta, \mathbf{z})\right) \\
& +(p-q) \sum_{i=1}^{N} \alpha\left(z_{i} ; \mathbf{z}\right)\left(1-\zeta\left(z_{i}-1 ; \mathbf{z}\right)\right)\left(f\left(\eta, \mathbf{z}^{\mathbf{z}_{i}, z_{i}-1}\right)-f(\eta, \mathbf{z})\right) \\
& +(p-q) \sum_{i=1}^{N} \eta\left(z_{i}+1\right)\left(f\left(\eta^{z_{i}+1, z_{i}}, \mathbf{z}^{z_{i}, z_{i}+1}\right)-f(\eta, \mathbf{z})\right) \\
& +q \sum_{u}\left(f\left(\eta_{u, u+1}, \mathbf{z}^{u, u+1}\right)-f(\eta, \mathbf{z})\right)
\end{aligned}
$$

where $\mathbf{z}^{u, v}$ is the configuration obtained from $\mathbf{z}$ by exchanging the content of $u$ with the content of $v$. For example if $z_{i}=u$ and $z_{j}=v$ for some $i, j(i \neq j)$, then in $\mathbf{z}^{u, v}$, the $i$-th particle is sitting at $v$ and the $j$-th particle is sitting at $u$.

Step 4. - We already know how $\alpha(u)=\zeta(u)-\eta(u)$ are distributed initially. We assume that $\mathbf{z}$ is distributed symmetrically at $t=0$. In other words, if at $t=0$, we change the labels the probability assigned to a configuration does not change. This property survives at later times. We write

$$
\mathbf{z}_{t}=\left(z_{1, t}, \ldots, z_{N, t}\right)
$$


and we claim that there is a coupling of the processes $\eta, \mathbf{z}, x$ such that if $z_{1,0} \geq x_{0}$ then

$$
z_{1, t} \geq x_{t}
$$

for all $t$. To see such coupling exists, we compare the rate of the jump of $x_{t}$ with $z_{1, t}$.

Suppose for some $t, x_{t}=z_{1, t}=u$. Then

(1) $x_{t}$ jumps to the left with rate $p-q$ if $\eta_{t}(u-1)=0$ and

(2) $x_{t}$ jumps to the left with rate $q$

while

(1) $z_{1, t}$ jumps to the left with rate $p-q$ if $\zeta_{t}(u-1)=0$ and

(2) $z_{1, t}$ jumps to the left with rate $q$.

Therefore the rate of the jump to the left for $x_{t}$ is not less than the rate for $z_{t, 1}$ because $\zeta(u)=0$ implies $\eta(u)=0$. Moreover,

(1) $x_{t}$ jumps to the right with rate $p-q$ if $\eta(u+1)=1$ and

(2) $x_{t}$ jumps to the right with rate $q$

while,

(1) $z_{1, t}$ jumps to the right with rate $p-q$ if $\eta(u+1)=1$ and

(2) $z_{1, t}$ jumps to the right with rate $q$.

Again we conclude that the rate of the jump to the right for $x_{t}$ is not greater than the rate for $z_{1, t}$. From this and the previous discussion we conclude that there is a coupling of $x_{t}$ and $z_{1, t}$ such that $x_{t} \leq z_{1, t}$ for all times. We omit the tedious formula of the generator of the coupled process.

Step 5. - Fix a positive $\varepsilon$. Let $\rho_{\delta}^{ \pm}(x, t)$ be such that initially $\rho_{\delta}^{ \pm}$ equals $\rho_{0, \delta}$. By Theorem 2.11 there exists a $\delta=\delta(\varepsilon)$ such that $\rho_{\delta}^{ \pm}(x, t)=\rho(x, t)$ for $t \in[0, T]$ if $(x, t)$ does not belong to the set $\Gamma_{\varepsilon}=\left\{(x, t): x^{-}(a, t)-\varepsilon \leq x \leq x^{+}(a, t)+\varepsilon, 0 \leq t \leq T\right\}$. Let $R_{L}$ denote the law of the $(x, \mathbf{z})$ process. Then by Theorem 2.2 applied to both the $\eta$ and $\zeta$ process, we have

$$
\lim _{L \rightarrow \infty} E^{R_{L}} \frac{1}{L} \sum_{i=1}^{N} J\left(\frac{1}{L} z_{i, L t}, t\right)=\int J(x, t)\left(\rho_{\delta}(x, t)-\rho(x, t)\right) d x .
$$

In particular, if $J=\mathbb{1}_{\Gamma_{\varepsilon}^{c}}$, the indicator function of the set $\Gamma_{\varepsilon}^{c}$, we obtain

$$
\lim _{L \rightarrow \infty} E^{R_{L}} \frac{1}{L} \sum_{i=1}^{N} \mathbb{1}_{\Gamma_{\varepsilon}^{c}}\left(\frac{1}{L} z_{i, L t}, t\right)=0 .
$$


Here $\Gamma_{\varepsilon}^{c}$ denotes the complement of the set $\Gamma_{\varepsilon}$. On the other hand, by symmetry assumption (see the first paragraph of Step 4),

$$
R_{L}\left(\left(\frac{1}{L} z_{1, L t}, t\right) \in \Gamma_{\varepsilon}^{c}\right)=E^{R_{L}} \frac{1}{N} \sum_{i=1}^{N} \mathbb{1}_{\Gamma_{\varepsilon}^{c}}\left(\frac{1}{L} z_{i, L t}, t\right) .
$$

Moreover,

$$
\lim _{L \rightarrow \infty} \frac{N}{L}=\int \rho_{\delta}(x, t) d x-\int \rho(x, t) d x \neq 0
$$

which follows from our assumption 2.19. This, (4.7) and (4.6) imply

$$
\lim _{L \rightarrow \infty} R_{L}\left(\left(\frac{1}{L} z_{1, t L}, t\right) \in \Gamma_{\varepsilon}^{c}\right)=0
$$

for every $t$.

Since $x_{t} \leq z_{1, t}$ we have

$$
\lim _{L \rightarrow \infty} R_{L}\left(\frac{1}{L} x_{t L} \leq x^{+}(a, t)+\varepsilon \text { for all } t \in A\right)=1
$$

where $A$ is any finite subset of $[0, T]$.

Final Step. - Let $\xi_{t}$ be a process generated by $\mathcal{L}$ with initial distribution $\mu_{L}^{\delta} \sim \rho_{0, \delta}$ where

$$
\rho_{0, \delta}(x)= \begin{cases}0 & \text { if } x \in[a-\delta, a] \\ \rho_{0}(x) & \text { otherwise }\end{cases}
$$

By monotonicity we can write

$$
\zeta_{t}=\eta_{t}-\beta_{t}, \quad \beta_{t} \in\{0,1\}^{\mathbb{Z}} .
$$

As in the previous step, it is possible to couple the processes $\beta_{t}, x_{t}$ so that a good number of the $\beta$-particles stay on the left of $x_{t}$ for all times. In the same way we conclude that for every positive $\varepsilon$,

$$
\lim _{L \rightarrow \infty} R_{L}\left(\frac{1}{L} x_{t L} \geq x^{-}(a, t)-\varepsilon \text { for all } t \in A\right)=1,
$$

where $A$ is any finite subset of $[0, T]$. Thus

$$
\lim _{L \rightarrow \infty} R_{L}\left(x^{-}(a, t)-\varepsilon \leq \frac{1}{L} x_{t L} \leq x^{+}(a, t)+\varepsilon \text { for all } t \in A\right)=1
$$

which implies that any limit point of $Q_{L}$ concentrated on the trajectory of the unique solution of (2.17) providing (2.18) holds. 
Remark 4.1. - If (2.18) does not hold we can still prove that any limit point of $\left\{Q_{L}\right\}$ is concentrated on the set of all solutions of (2.17). We know that if (2.18) fails we may have more than one solution.

We follow the same strategy for the proof of Theorem 2.10. The following formulation of the concavity of $g$ will make it possible to compare the jump rate of $z_{1, t}$ with $x_{t}$ in ZRP.

We omit the easy proof of the following lemma.

LEMMA 4.2. - The concavity of $g$ is equivalent to

$$
\begin{aligned}
\frac{g(n+k)-g(n)}{k} \leq & g(n+1)-g(n) \leq \frac{g(n)-g\left(n-k^{\prime}\right)}{k^{\prime}} \\
& k>0, k^{\prime} \in(0, n] .
\end{aligned}
$$

Proof of Theorem 2.10. - In this case $\mathcal{A}$ can be written as

$$
\mathcal{A} f(\eta, x)=\mathcal{L} f(\eta, x)+\sum_{u}(g(\eta(u)+1)-g(\eta(u)))(f(\eta, x+1)-f(\eta, x))
$$

where $\mathcal{L}$ acts on $\eta$ variables only.

Suppose $\zeta$ is as in the second step of the previous proof,

$$
\zeta=\eta+\alpha
$$

The generator of the process $(\eta, \alpha)$ can be written as

$$
\mathcal{B} f(\eta, \alpha)=\mathcal{L} f(\eta, \alpha)+\sum_{u}(g(\eta(u)+\alpha(u))-g(\eta(u)))\left(f\left(\eta, \alpha^{u, u+1}\right)-f(\eta, \alpha)\right)
$$

As in the third step of the previous proof we label $\alpha$-particles from 1 to $N$ where

$$
N=\sum_{u} \alpha(u)
$$

Let $\mathbf{z}=\left(z_{1} \ldots z_{N}\right)$ denote the locations of the $\alpha$-particles;

$$
\begin{aligned}
\alpha(u ; \mathbf{z}) & =\left\{\# i: z_{i}=u\right\} \\
\zeta(u ; \mathbf{z}) & =\eta(u)+\alpha(u ; \mathbf{z})
\end{aligned}
$$

The rate of the jump of an $\alpha$-particle is $g(n+k)-g(n)$ if $n=\eta(u)$ and $k=\alpha(u ; \mathbf{z})$. We assume that each $\alpha$-particle jumps with rate Vol. $12, n^{\circ} 2-1995$ 
$\frac{1}{k}(g(n+k)-g(n))$ so that the total rate of a jump from $u$ to $u+1$ becomes $g(n+k)-g(n)$. The generator of $(\eta, \mathbf{z})$ is

$\widehat{\mathcal{B}} f(\eta, \mathbf{z})=\mathcal{L} f(\eta, \mathbf{z})+\sum_{i} \frac{g\left(\zeta\left(z_{i} ; \mathbf{z}\right)\right)-g\left(\eta\left(z_{i}\right)\right)}{\alpha\left(z_{i} ; \mathbf{z}\right)}\left(f\left(\eta, \mathbf{z}^{z_{i}, z_{i}+1}\right)-f(\eta, \mathbf{z})\right)$

where $\mathbf{z}^{z_{i}, z_{i}+1}$ is a confiuration obtained from $\mathbf{z}$ by moving the $i$-th particle from $z_{i}$ to $z_{i}+1$.

The first inequality in (4.12) implies that the jump rate to the right for $x_{t}$ is not less than the jump rate for $z_{1, t}$. Therefore we can couple $x$ with $z_{1}$ in such a way that $z_{1, t}$ always stays on the left of $x_{t}$. This implies (4.8). The other inequality in (4.12) and (2.12) can be used to deduce (4.10).

It is also possible to prove something like Theorem 2.10 for processus misanthrope. The concavity assumption or equivalently (4.12), is replaced with

(i) $b(n+1, m)-b(n, m) \geq \frac{1}{\ell}(b(n+\ell, m)-b(n, m))$ for $\ell>0$,

(ii) $b(n, m)-b(n, m+1) \geq \frac{1}{k}(b(n, m)-b(n+\ell, m+k))$ whenever $b(n, m)>b(n+\ell, m+k)$,

(iii) $b(n+1, m)-b(n, m) \geq \frac{1}{\ell}(b(n, m)-b(n-\ell, m-k))$ whenever $b(n, m)>b(n-\ell, m-k)$ and $\ell \in(0, n], k \in[0, m]$.

(iv) $b(n, m)-b(n, m+1) \geq \frac{1}{\ell}(b(n, m-\ell)-b(n, m))$ for $\ell \in(0, m]$.

These conditions imply (but are not equivalent to) the concavity of $b(n, m)$ in $n$ and $m$.

\section{FLUCTUATIONS}

It is expected that both the macroscopic density and the location of the shock undergo Gaussian fluctuations.

For simplicity we assume $\rho$ is smooth in the set $[0, T] \times \mathbb{R}$ except on the shock curve

$$
\{(t, x(t)): 0 \leq t \leq T\}
$$

with $x(0)=a$. 
To model the fluctuation we write

$$
\eta_{t L}([x L])=m(x, t)+o\left(\frac{1}{\sqrt{L}}\right) .
$$

We expect that for a suitable (random) function $\hat{x}(t)$,

$$
m(x, t)= \begin{cases}\rho^{-}(x, t)+O\left(\frac{1}{\sqrt{L}}\right) & \text { if } x(t)<\hat{x}(t) \text { and } x \in[x(t), \hat{x}(t)] \\ \rho^{+}(x, t)+O\left(\frac{1}{\sqrt{L}}\right) & \text { if } \hat{x}(t)<x(t) \text { and } x \in[\hat{x}(t), x(t)]\end{cases}
$$

We regard $\hat{x}(t)$ as the "true" location of the shock.

We conjecture that the position of the second class particle $\frac{1}{L} x_{t L}$ is approximated by $\hat{x}(t)$ with an error of order $o\left(\frac{1}{\sqrt{L}}\right)$.

If $x$ is not between $x(t)$ and $\hat{x}(t)$, then we expect

$$
m(x, t)=\rho(x, t)+\frac{1}{\sqrt{L}} \xi(x, t)
$$

where $\xi$ is the fluctuation in the density and it satisfies the linearized hydrodynamics equation

$$
\xi_{t}+\left(\gamma h^{\prime}(\rho) \xi\right)_{x}=0 .
$$

Initially $\xi(x, 0)=\xi_{0}(x)$ is a Gaussian white noise that in the case of SEP its covariance is given by

$$
\left\langle\xi_{0}(x) \xi_{0}(y)\right\rangle=\delta(x-y) \rho_{0}(x)\left(1-\rho_{0}(x)\right) .
$$

See for example [14], part II, chapter 5.

Fix $t$ and let $\omega^{ \pm}(s)=y^{ \pm}(x(t) ; s, t)$ for $0 \leq x \leq t$. It follows from Lemma 3.6 that $\omega^{+}$and $\omega^{-}$are characteristic lines.

Since on the set

$$
\{(x, t): x<x(t), 0<t<T\}
$$

$\rho$ is smooth, it is not hard to show that a solution $\xi$ to (5.4) satisfies

$$
\int_{-\infty}^{z(t)} \xi(z, t) d z=\int_{-\infty}^{z(0)} \xi_{0}(z) d z
$$


for every characteristic line $z(t)$. This implies

$$
\int_{-\infty}^{\omega^{-}(t)} \xi(z, t) d z=\int_{-\infty}^{\omega^{-}(0)} \xi_{0}(z) d z
$$

Similarly,

$$
\int_{\omega^{+}(t)}^{\infty} \xi(z, t) d z=\int_{\omega^{+}(0)}^{\infty} \xi_{0}(z) d z
$$

Therefore

$$
\int_{-\infty}^{\infty} \xi(z, t) d z=\int_{-\infty}^{\infty} \xi_{0}(z) d z-\int_{y^{-}(x, t)}^{y^{+}(x, t)} \xi_{0}(z) d z
$$

because $\omega^{ \pm}(0)=y^{ \pm}(x, t)$ and $\omega^{ \pm}(t)=x$.

Now we argue that the second term in the right-hand side of (5.5) is responsible for the fluctuation in the location of the shock.

Since the total number of particles is conserved, we have

$$
\int_{-\infty}^{\infty} m(z, t) d z=\int_{-\infty}^{\infty} m(z, 0) d z+o\left(\frac{1}{\sqrt{L}}\right) .
$$

On the other hand,

$$
\begin{aligned}
\int_{-\infty}^{\infty} m(z, t) d z & =\frac{1}{\sqrt{L}} \int_{-\infty}^{\infty} \xi(z, t)+\int_{-\infty}^{\infty} \rho(z, t) d z \\
& +\frac{1}{\sqrt{L}}(\hat{x}(t)-x(t))\left(\rho^{+}(x(t), t)-\rho^{-}(x(t), t)\right)+o\left(\frac{1}{\sqrt{L}}\right)
\end{aligned}
$$

using (5.2) and (5.3). This and (5.5) imply

$$
\hat{x}(t)-x(t)=\frac{1}{\sqrt{L}} \frac{1}{\rho^{+}(x(t), t)-\rho^{-}(x(t), t)} \int_{y^{-}(x(t), t)}^{y^{+}(x(t), t)} \xi_{0}(z) d z .
$$

In the case of SEP with $\gamma>0$ and if $\rho$ is a Riemann solution,

$$
\begin{gathered}
\rho(x, t)= \begin{cases}k^{-} & x<x(t) \\
k^{+} & x>x(t)\end{cases} \\
x(t)=\gamma\left(1-k^{-}-k^{+}\right) t
\end{gathered}
$$

with $k^{-}<k^{+}$, the derivation of (1.7) is due to Spohn [14]. 
A rigorous derivation of (1.7) can be found in [3] and [8] under the additional assumption $k^{-}=0$. The case $k^{-}>0$ has been recently treated by Ferrari and Fontes $[5,6]$.

In the case of ZRP, (1.7) was established by Wick [15] under the assumptions $p(1)=1, g(n)=\mathbb{1}(n>0)$, and $k^{-}=0$.

\section{ACKNOWLEDGEMENT}

I would like to thank the referee for his useful remarks on the original version of this article.

\section{REFERENCES}

[1] C. T. Cocozza, Processus des misanthropes, Z. Wahrs. Verw. Gebiete, Vol. 70, 1985, pp. 509-523.

[2] C. M. DAfERmos, Generalized characteristics and the structure of solutions of hyperbolic conservation laws, Indiana U. Math. J., Vol. 26, 1977, pp. 1097-1119.

[3] A. DeMasi, C. Kipnis, E. Presutti and E. Sadda, Microscopic structure at the shock in the asymmetric simple exclusion, Stochastics, Vol. 27, 1989, pp. 151-165.

[4] P. A. Ferrari, Shock fluctuations in asymmetric simple exclusions, Probab. Theor. Related Fields, Vol. 91, 1992, pp. 81-101.

[5] P. A. Ferrari and L. R. G. Fontes, Current fluctuations for the asymmetric simple exclusion process, preprint.

[6] P. A. Ferrari, and L. R. G. Fontes, Shock fluctuations in the asymmetric simple exclusino process, preprint.

[7] A. F. FIlipPov, Differential equations with discontinuous right-hand side, Mat. Sbornik (N.S.), Vol. 51 (93), 1960, pp. 93-128.

[8] J. GÄRTner and E. Presutti, Shock fluctuations in a particle system, Annales de l'I.H.P., Vol. 53, 1989, pp. 1-14.

[9] P. D. LAx, Hyperbolic systems of conservation laws and the mathematical theory of shock waves, Conf. Board Math. Sci., Vol. 11, SIAM, 1973.

[10] P. D. LAX, Hyperbolic systems of conservation laws, Commun. Pure Appl. Math., Vol. 10, 1957, pp. 537-566.

[11] T. M. LiggetT, Interacting Particle Systems, Springer, 1985.

[12] F. Rezakhanlou, Evolution of tagged particles in non-reversible particle systems, to appear in Commun. Math. Phys.

[13] F. Rezakhanlou, Hydrodynamic limit for attractive particle systems on $\mathbb{Z}^{d}$, Commun. Math. Phys., Vol. 140, 1991, pp. 417-448.

[14] H. SPOHN, Large Scale Dynamics for Interacting Particles, Springer, 1991.

[15] W. D. WICk, A dynamical phase transition in an infinite particle system, J. Stat. Phys., Vol. 38, 1985, pp. 1015-1025. 